\title{
Atomic-Scale Mapping of Thermoelectric Power on Graphene: Role of Defects and Boundaries
}

\author{
Jewook Park ${ }^{1}$, Guowei $\mathrm{He}^{2}$, R. M. Feenstra ${ }^{2}$, and An-Ping $\mathrm{Li}^{1}$ \\ ${ }^{1}$ Center for Nanophase Materials Sciences, Oak Ridge National Laboratory, Oak Ridge, \\ Tennessee 37831, USA \\ ${ }^{2}$ Department of Physics, Carnegie Mellon University, Pittsburgh, PA 15213, USA
}

\begin{abstract}
The spatially resolved thermoelectric power is studied on epitaxial graphene on $\mathrm{SiC}$ with direct correspondence to graphene atomic structures by a scanning tunneling microscopy (STM) method. A thermovoltage arises from a temperature gradient between the STM tip and the sample, and variations of thermovoltage are distinguished at defects and boundaries with atomic resolution. The epitaxial graphene has a high thermoelectric power of $42 \mu \mathrm{V} / \mathrm{K}$ with a big change $(9.6 \mu \mathrm{V} / \mathrm{K})$ at the monolayer-bilayer boundary. Long-wavelength oscillations are revealed in thermopower maps which correspond to the Friedel oscillations of electronic density of states associated with the intravalley scattering in graphene. On the same terrace of a graphene layer, thermopower distributions show domain structures that can be attributed to the modifications of local electronic structures induced by microscopic distortions (wrinkles) of graphene sheet on the $\mathrm{SiC}$ substrate. The thermoelectric power, the electronic structure, the carrier concentration, and their interplay are analyzed on the level of individual defects and boundaries in graphene.
\end{abstract}

\section{Introduction}

Graphene, a single layer of graphite, has outstanding electronic and transport properties associated with its unique band structures. The symmetry of the graphene honeycomb lattice is a key element for determining graphene's electronic properties. ${ }^{1}$ However, defects and boundaries $^{2-6}$ can break the sublattice symmetry and are thought to play a major role in the electronic scattering processes in the graphene sheets. ${ }^{7-9}$ Extensive electrical transport studies have been performed to understand the defect scattering in graphene. ${ }^{10-13}$ Compared with electrical transport, the thermoelectric properties provide complementary information to the electronic structures and the detail of electron scattering. In particular, the thermoelectric power is of great interest due to its extreme sensitivity to the particle-hole asymmetry of a system. ${ }^{14}$ Measurement of the thermoelectric power of graphene can thus elucidate details of the electronic structure of the ambipolar nature of graphene that cannot be probed by conductance measurements alone. Indeed, the thermoelectric properties of this two-dimensional material have recently been extensively studied for revealing the intrinsic properties of Dirac electrons and elucidating effects of defects on its unique electronic structures. ${ }^{14-21}$ In those studies, thermoelectric measurements are usually carried out with a technique developed by Kim et al. ${ }^{22}$ In this technique, a local heater made of a metal line produces a temperature difference $\Delta T$ between the two ends of a sample, which gives rise to a thermoelectric voltage $V_{t h}$ measured by electrodes defined by standard electron beam lithography and nanofabrication process. The thermopower is obtained as the ratio of $V_{t h}$ to $\Delta T$ across a sample. As a versatile technique for direct measurements of thermoelectric parameters, this method however lacks the spatial resolution for probing the variations of thermoelectric properties across a sample surface which 
can be the key for revealing the inhomogeneities and defect scattering effects in thermoelectric materials.

An alternative way to study the thermoelectric properties is to measure the thermovoltage $V_{t h}$ between a tunneling tip and a sample caused by a $\Delta T$ across the tunneling barrier of a scanning tunneling microscope (STM). This method was first applied by Williams and Wickramasinghe ${ }^{23}$ for $\mathrm{MoS}_{2}$, and Moeller et al. ${ }^{24-28}$ extended it to a wide variety of applications. The thermovoltage is very sensitive to the local density of electronic states and the chemical potential variations. In particular, a thermoelectric power profile were measured across semiconductor interfaces, ${ }^{29}$ molecular junctions, ${ }^{30}$ and metallic domain boundaries ${ }^{26-28}$ in nanoscopic resolution. Here we use the STM to examine the thermovoltage distributions down to atomic resolution in single and bilayer graphene films epitaxially grown on the silicon terminated $\mathrm{SiC}$ surface. We report on quantitative values for the thermoelectric power difference across boundaries defined by graphene thickness change and demonstrate how local structural defects can profoundly change the thermoelectric power. The relationships between structure and thermoelectric properties are evaluated at the atomic scale.

\section{Experimental}

The measurement of thermovoltage with STM can be understood in the following way. As the STM tip and sample come within tunneling range, their electronic states become strongly coupled and their chemical potentials tend to equalize by two-way electron tunneling across the gap. If there is a temperature gradient in the tunneling-junction and the sample surface is conductive enough, a thermovoltage will be set up across the tip-sample junction. Stovneng and Lipavský ${ }^{31}$ showed that, if the local density of states varies approximately linearly as a function of energy around the Fermi level, the thermovoltage can be approximated by

$$
V_{t h}=\frac{\pi^{2} k^{2}}{6 e_{0}}\left(T_{t}^{2}-T_{s}^{2}\right)\left(\frac{\partial \ln \rho_{t}}{\partial E}+\frac{\partial \ln \rho_{s}(x, y)}{\partial E}+\frac{z}{\hbar} \sqrt{\frac{2 m}{\phi}}\right),
$$

where $k$ is the Boltzmann's constant, $\hbar$ is Planck's constant, $m$ is the electron mass, and $\phi$ is the work function, $T_{t}, T_{s}$ are the temperatures of tip and sample, $\rho_{t}, \rho_{s}(x, y)$ are the density of electronic states at the Fermi level, $E$ is the energy, $z$ is the tip-sample distance. The thermovoltage is given by the sum of the logarithmic derivatives of the electronic density of states of the tip and sample and a third term containing the gap width and the work function. The corresponding thermopower $S$ can be obtained by

$$
S=\frac{V_{t h}}{T_{t}-T_{s}}=\frac{\pi^{2} k^{2}}{6 e_{0}}\left(T_{t}+T_{s}\right)\left(\frac{\partial \ln \rho_{t}}{\partial E}+\frac{\partial \ln \rho_{s}(x, y)}{\partial E}+\frac{z}{\hbar} \sqrt{\frac{2 m}{\phi}}\right)
$$

Experimentally, the thermovoltage is measured at open loop condition by a technique similar to the scanning tunneling potentiometry. ${ }^{32,33}$ Figure la shows a schematic diagram of the method to measure thermovoltage and topography simultaneously. The feedback loop for distance regulation makes use of an ac modulation of the bias voltage leading to an ac component of the tunneling current proportional to the tunneling conductance. Using an appropriate demodulation, it can be used for the adjustment of the distance between the tip and the sample. With a modified Nanonis Controlling Electronics, we can also benefit from the thermal noise of the tunneling junction providing an internal "modulation" with white noise. The power spectral density of this current noise is proportional to the tunneling conductance, hence it has the same exponential dependence on the distance between tip and sample and can be used instead of the tunneling current itself in the feedback loop for $z$ control. ${ }^{28}$ As explained by 
Moeller et al., ${ }^{26,34}$ the advantage of this technique is the absence of a "false" signal due to capacitive cross-talk which is always present when using an external modulation. A second feedback loop can be used to adjust the bias voltage such that the dc component of the tunneling current becomes zero. The required offset voltage corresponds to the thermovoltage of the tunneling barrier. In the variable temperature STM (Omicron VT-STM), the sample can be heated or cooled by several hundred $\mathrm{K}$ while the tip is maintained at room temperature. Since in vacuum the thermal coupling between tip and sample is negligible compared to the thermal conductance of tip and sample, ${ }^{35}$ the temperature difference is constant during the experiment, i.e., it does not vary while scanning.

\section{Results and Discussion}

First, we measure the thermovoltage on monolayer graphene grown on a $\mathrm{SiC}(0001)$ substrate. $^{36,37}$ Figure $1 \mathrm{~b}$ shows the STM image of the monolayer graphene measured at $130 \mathrm{~K}$, where graphene surface is dominated by the $(6 \times 6)$ periodicity coming from the buffer layer but atomic structures of honeycomb lattice can still be resolved. Figure 1c is the thermovoltage distribution map acquired simultaneously with the topography. An averaged thermovoltage $V_{t h}$ is obtained to be about $7.12 \mathrm{mV}$ with a temperature gradient $\Delta T=168.5 \mathrm{~K}$. The corresponding thermopower $S=42 \mu \mathrm{V} / \mathrm{K}$. In comparison, depending on the carrier concentrations, thermopower values of graphene have been reported to reach $80 \mu \mathrm{V} / \mathrm{K}^{14,15,38}$ as measured with conventional microfabricated heater and thermometer electrodes, much higher than the value in a 2D GaAs/AlGaAs heterojunction. Note, the sign of thermopower in STM measurement does not reflect the type of majority carriers, and it instead corresponds to the logarithmic derivatives of the electronic density of states as explained in Eq. (2). Interestingly, the buffer layer interference has a smaller influence on the thermovoltage than it does on the topography. Atomic resolution thermovoltage distribution is observed, which is not because of the large temperature difference across the vacuum gap, but due to the strong contribution of the sample local density of states (LDOS) to the thermovoltage.

We then examine the thermovoltage profiles across a graphene boundary, defined by changes in layer thickness and substrate steps in epitaxial graphene. Figure 2a shows the STM image of a boundary between monolayer (ML) and bilayer (BL) graphene. As a result of graphitization of $\mathrm{SiC}(0001)$ in the epitaxial graphene growth process, a ML-BL boundary almost always coincides with an underlying substrate step. ${ }^{6}$ STM images on both sides of the step show that the lattice structure of graphene surface remains unchanged across the boundary, namely a carpet-like growth mode covering the substrate step and terraces. Figure $2 \mathrm{~b}$ is a schematic structure of the boundary, where on the left side is a ML graphene atop the $6 \sqrt{3} \times 6 \sqrt{3}) R 30^{\circ}$ buffer layer and on the right is a BL of graphene atop a buffer layer depressed by three $\mathrm{SiC}$ substrate layers. Figure $2 \mathrm{c}$ shows a height profile of the ML-BL boundary, where the upper terrace is $\sim 4.0 \AA$ higher than the lower terrace, in good agreement with the expectation of subtracting the interlayer spacing of two graphene sheets $(3.3 \AA)$ from the three interlayer spacings of $\mathrm{SiC}(3 \times 2.5 \AA){ }^{39,40}$. Figure $2 \mathrm{~d}$ shows the thermovoltage profile across the boundary measured when the sample temperature is $310 \mathrm{~K}$ while the tip remains at room temperature $(298.5 \mathrm{~K})$ with $\Delta T=-11.5 \mathrm{~K}$. A higher thermovoltage is seen on the lower terrace of BL graphene than on upper terrace of the ML, showing a thermopower difference of $9.6 \mu \mathrm{V} / \mathrm{K}$ across the boundary.

In comparison, we find the thermovoltage vanishes when the sample is measured at the same temperature as the tip. The inset of Fig $2 \mathrm{~d}$ shows the measurements on the same ML-BL 
boundary at room temperature $(298.5 \mathrm{~K})$ with $\Delta T=0 \mathrm{~K}$, where the averaged $V_{\text {th }}$ is nearly zero and remains essentially unchanged across the boundary. These results thus confirm that the thermovoltage measured with STM does not come from the cross-talk of STM image and it directly reflects the thermoelectric response associated with the electronic density of states in the material.

Thermovoltage distribution is very sensitive to defect scattering. In Fig. 3, a ML-BL boundary is shown with topography (Fig. 3a) and thermovoltage (Fig. 3b) measured at $130 \mathrm{~K}$ $(\Delta T=168.5 \mathrm{~K})$. The line profiles (Fig. 3c) show that thermovoltage of ML is higher than that of $\mathrm{BL}$, though the ML terrace is lower in $z$. Also, the thermovoltage displays a strong upturn at the transition region $(\sim 10 \mathrm{~nm})$ of the boundary. This is well consistent with our earlier observation of a Fermi level shift toward the Dirac point near the step edges. ${ }^{4}$ Indeed, as proposed by Low $e t$ al., ${ }^{6}$ the carrier concentration can vary at the step edge due to increased distance and suppressed interactions between graphene and $\mathrm{SiC}$. The Fermi level shift changes the local density of states and thus the thermovoltage at the step edge. Away from the step edge where the carrier concentrations are believed to be the same on both sides of the boundary, the thermovoltage distributions correspond to the variations of the density of states on both terraces.

Moreover, the thermovoltage map shows some wavy structures near the step edge and point defect-like dark spots on terraces, features that are not obvious in the corresponding STM image. As shown in Fig. 3d, the wavy structure has a clear oscillatory nature with a period of 6.4 $\mathrm{nm}$ near the step edge. The largest modulation is found on the BL terrace near the step edge, and a closer look reveals that it is also present in the topography image. This observation of longwavelength oscillations resembles the findings on $\mathrm{BL}$ graphene on SiC by Rutter et al., corresponding to the Friedel oscillations associated to the intravalley scattering processes in graphene. A faster decay of the oscillations than in Fermi liquids is direct consequence of the chiral nature of the quasiparticles in graphene. ${ }^{42}$ Interestingly, the long-wavelength Friedel oscillations are not obvious on the ML graphene due to the suppression of intravalley scattering. ${ }^{43}$

Strikingly, we find an unusual domain structure in the thermovoltage map that extends over the ML-BL graphene boundary. Figure 4a shows the large scale topography of a ML-BL graphene boundary at $130 \mathrm{~K}$ with a clear terrace structure. However, the corresponding thermovoltage image (Fig. 4b) displays irregular domains that intersect with the graphene boundary. The three-dimensional contour images in Fig. 4c and d clearly demonstrate the difference between topography and thermovoltage across the boundary. The topography contour shows the BL on the left side of the boundary is higher than the ML on the right. The thermovoltage image, however, shows a lower $V_{\text {th }}$ on the BL side than the ML side, and on each side of the boundary there exist domains going over the step boundary. The clear modifications of thermovoltage indicate dramatic changes in the LDOS even on the same graphene terrace. To examine the LDOS variations, we map in Fig. 4e the tunneling $d I / d V$ spectroscopy data measured by STS, where a direct correspondence can be identified between the thermovoltage and $d I / d V$ in terms of domain structures. A few individual $d I / d V$ curves are displayed in Fig. 4f, where region 2 shows a clear local minimum at Dirac energy as well as higher conductivity than regions 1 and 3 .

A further evaluation of the domain structures is displayed in Fig. $4 \mathrm{~g}$ with the line profiles both for the height and thermovoltage along the same line on the BL terrace. A small protrusion $(2-3 \AA)$ is seen in $z$ profile at domain boundaries. The region 2 displays higher $d I / d V$ and lower $z$ height, and vice versa at region 1 and 3. Since the domain structure occurs locally regardless of 
the step boundary, we believe it comes from the structure distortions in the graphene layer that can form by the mismatch of the thermal expansion coefficient between graphene and SiC. Due to the negative thermal expansion coefficient of graphene, ${ }^{44}$ graphene expands during the cooling process from growth temperature, which can create wrinkles with long-range microscopic corrugations and lead to local detachment of graphene sheet from the underneath buffer layer, as schematically shown in Fig. 4h. The detached region has much weaker interactions with the buffer layer. The wrinkle can collapse back to the substrate but chemical bonds to the buffer layer are broken. Thus the topography does not show much $z$ change except at the domain edges but the electronic density of states is significantly modified in the collapsed wrinkle region. This is why the Dirac point can be seen in STS for region 2 but not for regions 1 and 3. The wrinkles go across the step edge and lead to the domains with different electronic properties and thermopower values.

\section{Conclusions}

We have measured the spatial distributions of thermovoltage that is induced by a temperature difference between tip and sample by using an STM on an epitaxial graphene on SiC. The thermovoltage has a direct correspondence to the electronic density of states, and defects and boundaries show clear impact to the thermoelectric response. A thermopower jump is observed at the monolayer and bilayer graphene boundary and the thermopower is modified by Friedel oscillations of the charge density in graphene. Beside the change at the ML-BL graphene boundary, the thermopower also provides spectroscopy maps which reveal domain structures induced by collapsed graphene wrinkles that not obvious in STM images. The thermopower distribution measurement with STM thus allows probing the electronic, thermoelectric, and structural properties down to the individual defect level.

\section{Methods}

STM Measurements

A summary of our experimental methods were published in a recent report. ${ }^{4}$ In brief, our experiments used an Omicro VT STM controlled by Electronics from Nanonis with a base pressure of $3 \times 10^{-11}$ Torr and electrochemically etched tungsten tips. Two feedback loops have been used to separate signals from topography and thermovoltage. The feedback loop for distance regulation makes use of the thermal noise of the tunneling junction for the adjustment of the distance between the tip and the sample. A second feedback loop can be used to adjust the bias voltage such that the dc component of the tunneling current becomes zero. The required offset voltage corresponds to the thermovoltage of the tunneling barrier. As a result, both topography and thermovoltage can be measured simultaneously at the same sample location.

\section{Graphene growth on $\mathrm{SiC}$}

The graphite heater, consisting of a bow-tie shaped graphite plate with $1 \mathrm{~mm}$ thickness and narrow neck measuring about $14 \mathrm{~mm}$ wide and $20 \mathrm{~mm}$ long. A $1 \mathrm{~cm} \times 1 \mathrm{~cm}$ sample rests on this narrow strip, and is heated by currents of typically 200 A passed through the strip. Watercooled copper clamps and electrical feedthroughs supply the current, and the heater is contained in an ultra-high-vacuum chamber. The $\mathrm{SiC}$ graphene growth procedure starts with hydrogen etching at $1620^{\circ} \mathrm{C}$ for $3 \mathrm{~min}$ followed by the graphene growth at $1590^{\circ} \mathrm{C}$ for 30 minutes in 1 atm argon environment. The graphene samples were grown on the $\mathrm{Si}$ face of $4 \mathrm{H}-\mathrm{SiC}$. The $\mathrm{Si}$ face of $\mathrm{SiC}$ allows for more controlled growth of the graphene thickness than the $\mathrm{C}$ face due to the fact 
that the Si face initially forms a C-rich buffer layer that acts as a template for the graphene formation as the $\mathrm{Si}$ is sublimed from the surface.

Acknowledgement. This research was conducted at the Center for Nanophase Materials Sciences, which is sponsored at Oak Ridge National Laboratory by the Office of Basic Energy Sciences, U.S. Department of Energy. The work at CMU was supported by the National Science Foundation.

1 P. L. McEuen, M. Bockrath, D. H. Cobden, Y.-G. Yoon, and S. G. Louie, Phys. Rev. Lett. 83, 5098 (1999).

2 C. Dimitrakopoulos, A. Grill, T. J. McArdle, Z. Liu, R. Wisnieff, and D. A. Antoniadis, Applied Physics Letters 98, 222105 (2011).

3 S. E. Bryan, Y. Yang, and R. Murali, The Journal of Physical Chemistry C 115, 10230 (2011).

4 K. W. Clark, X.-G. Zhang, I. V. Vlassiouk, G. He, R. M. Feenstra, and A.-P. Li, Unpublished (2013).

5 S.-H. Ji, J. B. Hannon, R. M. Tromp, V. Perebeinos, J. Tersoff, and F. M. Ross, Nat. Mater. 11, 114 (2012).

6 T. Low, V. Perebeinos, J. Tersoff, and P. Avouris, Phys. Rev. Lett. 108, 096601 (2012).

7 O. V. Yazyev and S. G. Louie, Physical Review B 81, 195420 (2010).

$8 \quad$ Y. Liu and B. I. Yakobson, Nano Letters 10, 2178 (2010).

9 Q. Yu, et al., Nat Mater 10, 443 (2011).

10 P. Y. Huang, et al., Nature 469, 389 (2010).

11 Q. Yu, et al., Nat. Mater. 10, 443 (2011).

12 J. C. Koepke, J. D. Wood, D. Estrada, Z.-Y. Ong, K. T. He, E. Pop, and J. W. Lyding, ACS Nano 7, 75 (2012).

13 J. D. Buron, et al., Nano Letters 12, 5074 (2012). Y. M. Zuev, W. Chang, and P. Kim, Physical Review Letters 102, 096807 (2009). X. Wu, Y. Hu, M. Ruan, N. K. Madiomanana, C. Berger, and W. A. d. Heer, Applied Physics Letters 99, 133102 (2011). M. S. Foster and I. L. Aleiner, Physical Review B 79, 085415 (2009).

H. Huang, W. Chen, S. Chen, D. C. Qi, X. Y. Gao, and A. T. S. Wee, Applied Physics Letters 94, 163304 (2009). P. Wei, W. Bao, Y. Pu, C. N. Lau, and J. Shi, Physical Review Letters 102, 166808 (2009).

J. G. Checkelsky and N. P. Ong, Physical Review B 80, 081413 (2009).

Z.-X. Xie, L.-M. Tang, C.-N. Pan, K.-M. Li, K.-Q. Chen, and W. Duan, Applied Physics Letters 100, 073105 (2012).

J. Y. Kim, J.-H. Lee, and J. C. Grossman, ACS Nano 6, 9050 (2012).

J. P. Small, K. M. Perez, and P. Kim, Physical Review Letters 91, 256801 (2003).

C. C. Williams and H. K. Wickramasinghe, Nature 344, 317 (1990).

D. Hoffmann, A. Haas, T. Kunstmann, J. Seifritz, and R. Moller, (AVS, 1997), p. 1418.

D. Hoffmann, J. Y. Grand, R. Möller, A. Rettenberger, and K. Läuger, Physical Review B 52, 13796 (1995).

D. Hoffmann, J. Seifritz, B. Weyers, and R. Möller, Journal of Electron Spectroscopy and Related Phenomena 109, 117 (2000). 
J. Seifritz, T. Wagner, B. Weyers, and R. Moller, Applied Physics Letters 94, 113112 (2009).

A. Rettenberger, C. Baur, K. Lauger, D. Hoffmann, J. Y. Grand, and R. Moller, Applied Physics Letters 67, 1217 (1995).

H.-K. Lyeo, A. A. Khajetoorians, L. Shi, K. P. Pipe, R. J. Ram, A. Shakouri, and C. K. Shih, Science 303, 816 (2004).

C. Evangeli, K. Gillemot, E. Leary, M. T. González, G. Rubio-Bollinger, C. J. Lambert, and N. Agrait, Nano Letters, ASAP (2013).

J. A. Sto/vneng and P. Lipavský, Physical Review B 42, 9214 (1990).

P. Muralt and D. W. Pohl, Appl. Phys. Lett. 48, 514 (1986).

A.-P. Li, K. W. Clark, X. G. Zhang, and A. P. Baddorf, Advanced Functional Materials, ASAP (2013).

J. Seifritz, T. Wagner, B. Weyers, and R. Moller, Applied Physics Letters 94, 113112 (2009).

J.-B. Xu, K. Lauger, R. Moller, K. Dransfeld, and I. H. Wilson, Journal of Applied Physics 76, 7209 (1994).

C. Berger, et al., J. Phys. Chem. B 108, 19912 (2004).

Luxmi, N. Srivastava, G. He, R. M. Feenstra, and P. J. Fisher, Phys. Rev. B 82, 235406 (2010).

A. N. Sidorov, et al., Applied Physics Letters 99, 013115 (2011).

T. Filleter, K. V. Emtsev, S. Th, and R. Bennewitz, Appl. Phys. Lett. 93, 133117 (2008).

P. Lauffer, K. V. Emtsev, R. Graupner, T. Seyller, and L. Ley, Phys. Rev. B 77, 155426 (2008).

G. M. Rutter, J. N. Crain, N. P. Guisinger, T. Li, P. N. First, and J. A. Stroscio, Science 317, 219 (2007).

J. Xue, J. Sanchez-Yamagishi, K. Watanabe, T. Taniguchi, P. Jarillo-Herrero, and B. J. LeRoy, Physical Review Letters 108, 016801 (2012).

P. Mallet, I. Brihuega, S. Bose, M. M. Ugeda, J. M. Gómez-Rodríguez, K. Kern, and J.

Y. Veuillen, Physical Review B 86, 045444 (2012).

D. Yoon, Y.-W. Son, and H. Cheong, Nano Letters 11, 3227 (2011). 
(a)

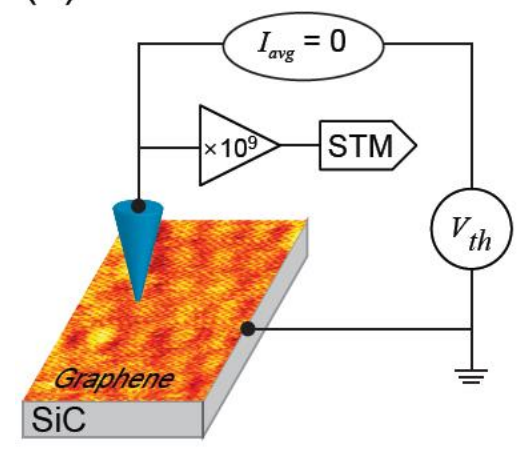

(b)

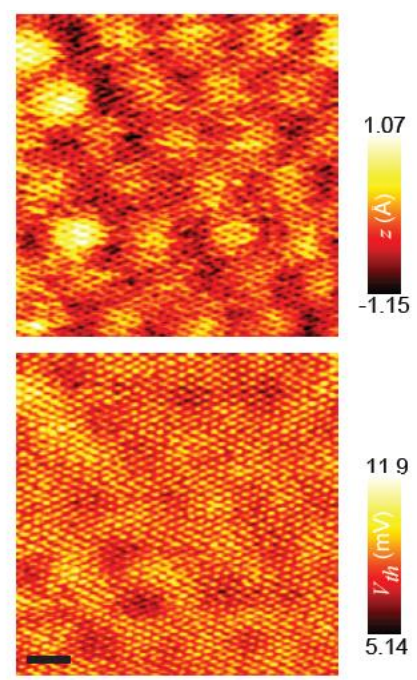

Figure 1. (Color online) Simultaneous structure and thermovoltage measurement with an STM at the atomic resolution on epitaxial graphene on SiC. (a). Schematic diagram of measurement technique. Atomic resolution images of topography (b) and thermovoltage (c) for the epitaxial graphene acquired simultaneously at $130 \mathrm{~K}$ (image size: $7.5 \mathrm{~nm} \times 7.5 \mathrm{~nm}$ ). The temperature at STM tip is $298.5 \mathrm{~K}$, with $\Delta T=168.5 \mathrm{~K}$.

(a)

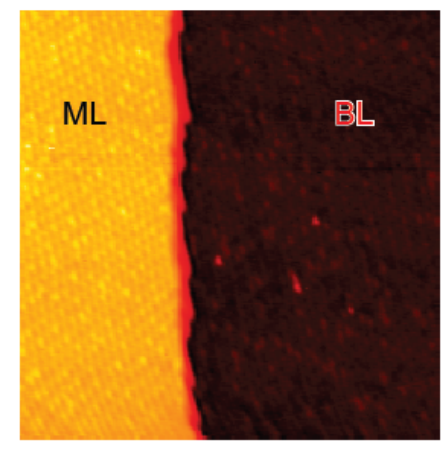

(c)

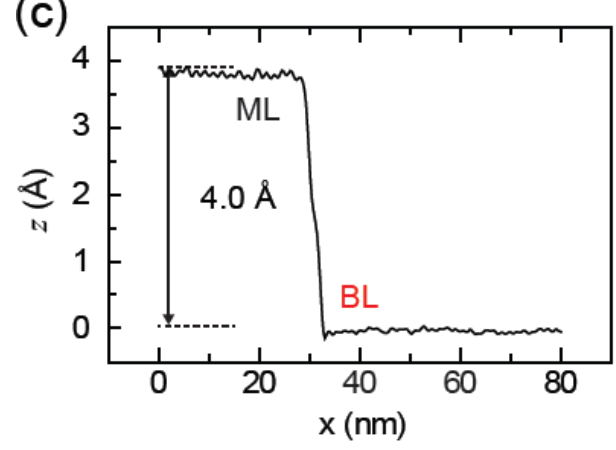

(b)

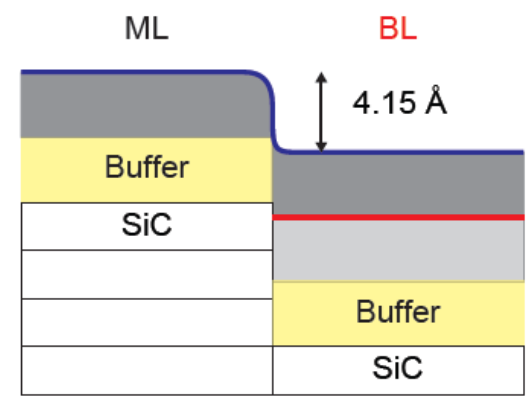

(d) 100

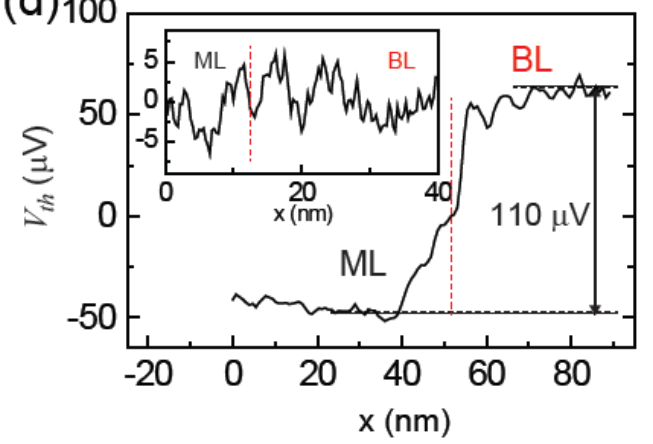

Figure 2. (Color online) Structure and thermovoltage at a ML-BL graphene boundary measured at $\mathrm{T}_{\text {sample }}=310 \mathrm{~K}$. (a). STM image of the ML-BL junction $(80 \mathrm{~nm} \times 80 \mathrm{~nm})$. (b). Schematic structure of the ML-BL boundary. Top graphene layer (blue line) seamlessly covered terrace step like a carpet. Light-blue line on BL terrace indicates the second graphene layer underneath the top layer. (c). Averaged $z$ height profile across the step. (d). Averaged thermovoltage line profile across the boundary with $\Delta T=-11.5 \mathrm{~K}$. The vertical red dashed line indicates the location of boundary. Inset: Thermovoltage across $\mathrm{ML}-\mathrm{BL}$ boundary at $\mathrm{T}_{\text {sample }}=298.5 \mathrm{~K}$ and $\Delta T=0 \mathrm{~K}$. 
(a)

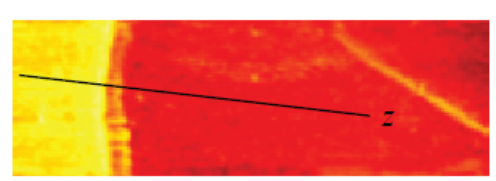

(b)

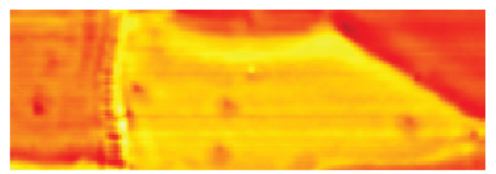

(c)

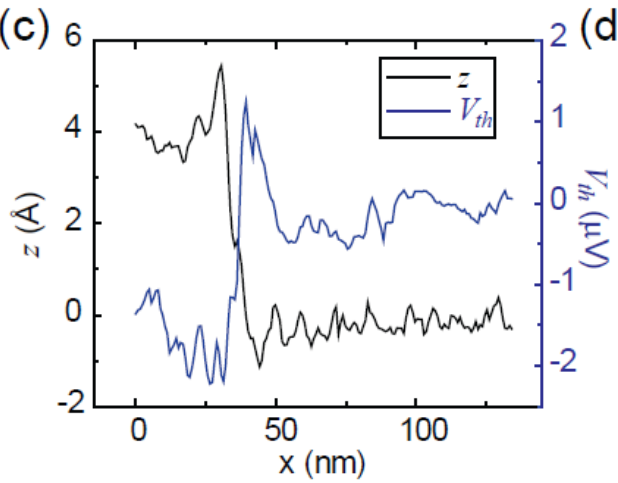

(d)

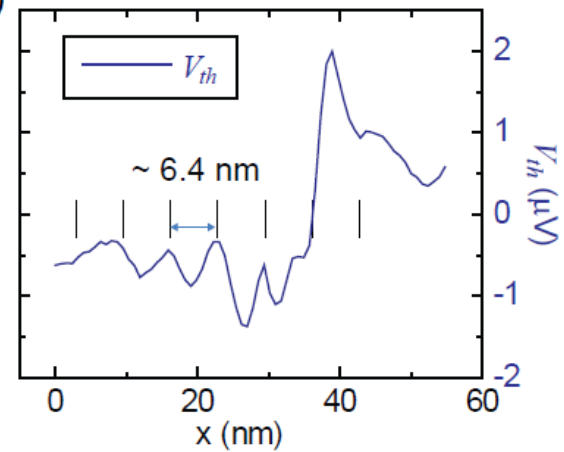

Figure 3. (Color online) Thermovoltage across a ML-BL graphene boundary. STM image (a) and thermovoltage image

(b) of the boundary measured at $\mathrm{T}_{\text {sample }}=130 \mathrm{~K}$. (c). The $z$ profile (black) and $V_{t h}$ profile (blue) measured simultaneously at the boundary. (d). Zoomed-in $V_{t h}$ map showing the oscillations near the step edge.

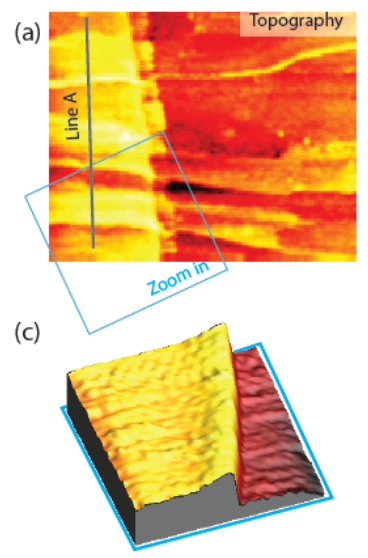

(d)

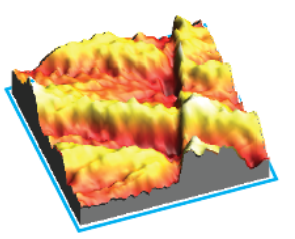

(i)

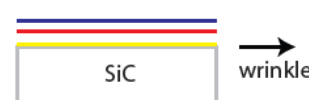

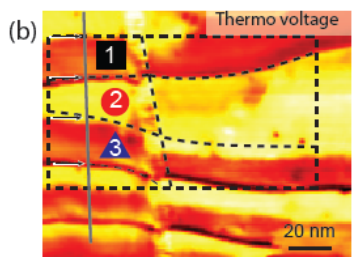
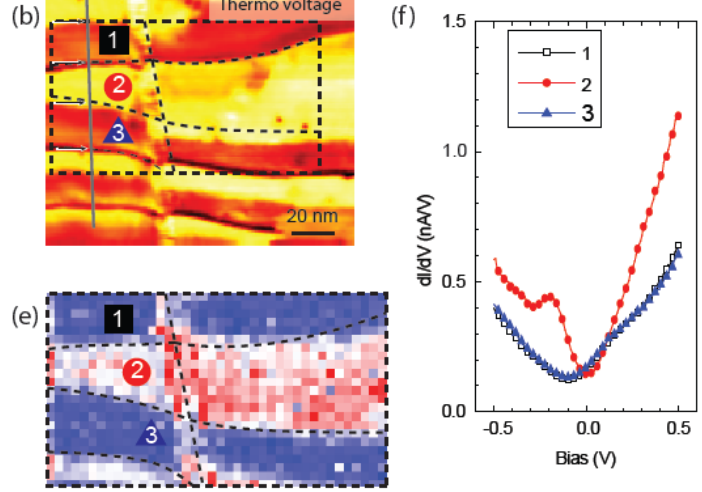

(g)
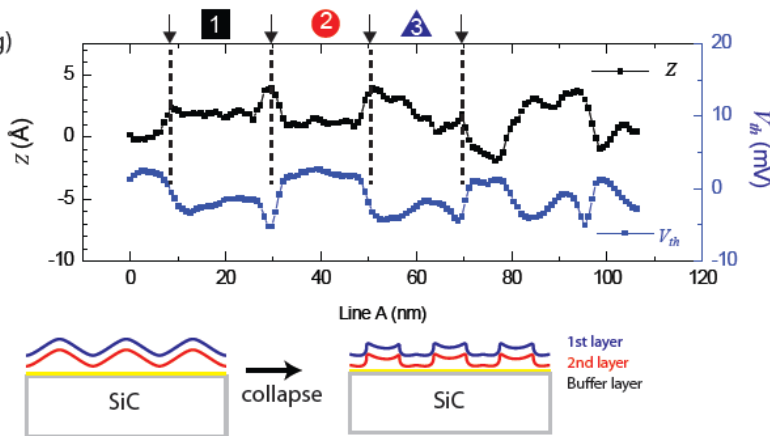

Figure 4. (Color online) Thermovoltage domains induced by structural wrinkles on epitaxial graphene. Simultaneously acquired topography (a) and thermovoltage image (b) at $130 \mathrm{~K}$. According to the measurements on the flat region of the same terrace structure (not shown here), the left and right side terraces are $\mathrm{BL}$ and ML, respectively. 3D contours of topography (c) and thermovoltage $(\mathrm{d})$ for

the region indicated by the square in (a). (e). Tunneling $d I / d V$ spectroscopy map at $+500 \mathrm{mV}$ for the region indicated in (a). (f). Area-averaged $d I / d V$ spectroscopy on different domains. (g). Line profile of the $z$ and $V_{t h}$ along a line indicated in (a). There is a height difference $(1 \sim 2 \AA)$ between domains despite the same terrace. Also, the gray arrows indicate the $z$ hump $(2 \sim 3 \AA)$ between domains. (h). Schematic diagrams for the wrinkled graphene layers. The thermal expansions create wrinkles on graphene layer and the wrinkled region collapses back to the buffer layer due to Van der Waals interaction leaving bumps at the wrinkle edges. 